\title{
Should 3D volume assessment of the corpus callosum and cerebellar vermis be a part of a routine second trimester screening?
}

\author{
Michaela Maderkova Tozzia , Jana Furstovab ${ }^{\mathrm{b}}$, Marek Lubusky ${ }^{\mathrm{a}}$
}

\begin{abstract}
Background. The majority of fetal structural defects can be detected in the second trimester, thus this is the main time for screening for structural defects. 3D imaging of the fetal brain does not create a common part of this screening. Methods. This prospective observational study was conducted at the Fetal Medicine Center of The GynecologicalObstetrical Department of the University Hospital Olomouc in years 2017-2020. The study sample was 451 consecutively scanned morphologically normal fetuses attending for routine second trimester anatomical survey at 20-22 weeks of pregnancy. A transabdominal 3D ultrasound volume acquisition of fetal brain was obtained from an axial and sagittal plane using skull sutures as an acoustic window.

Results. Both the corpus callosum (CC) and the vermis (VC) were detected in $51.7 \%$ of examinations in the sagittal plane, and in $31.7 \%$ in the axial plane. In $61.9 \%$ of the examinations, there was at least partial detection in both planes. Maternal BMI was found to be the only significant predictor of the quality of imaging in both planes.

Conclusion. 3D acquisition of fetal brain images in the sagittal plane followed by manipulation of acquired volume was valuable in assessment of corpus callosum and cerebellar vermis. This allows reconstruction of the sagittal plane that can be difficult to obtain in $2 \mathrm{D}$ imaging.
\end{abstract}

Key words: 3D ultrasound, central nervous system examination, prenatal diagnostics, corpus callosum, cerebellar vermis

Received: February 1, 2021; Revised: May 13, 2021; Accepted: May 13, 2021; Available online: May 27, 2021

https://doi.org/10.5507/bp.2021.033

(c) 2022 The Authors; https://creativecommons.org/licenses/by/4.0/

${ }^{a}$ Department of Obstetrics and Gynecology, Faculty of Medicine and Dentistry, Palacky University Olomouc and University Hospital Olomouc, Czech Republic

${ }^{b}$ Olomouc University Social Health Institute, Palacky University Olomouc, Czech Republic

Corresponding author: Michaela Maderkova Tozzi, e-mail: michaelatozzi@seznam.cz

\section{INTRODUCTION}

Central nervous system (CNS) defects are the most common of fetal structural defects ${ }^{1}$. Therefore ultrasound assessment of the fetal brain is a very important part of the screening for structural defects. The International Society of Ultrasound in Obstetrics and Gynecology (ISUOG) has published a Practice guideline ${ }^{1}$ for routine examination of the fetal brain during the second-trimester anomaly scan. It is based on a transabdominal evaluation of three axial planes through the fetal brain (transventricular, transthalamic, and the posteriorly tilted transcerebellar plane). These planes enable the evaluation of multiple structures, including the shape of the fetal head, midline falx, thalami, cavum septi pellucidi (CSP), lateral ventricles with choroids, cerebellum and cisterna magna, and permit fetal head biometry to be performed ${ }^{1}$.

The corpus callosum, a thick plate of dense myelinated fibers, is the main connection between the cerebral hemispheres. Its formation is initiated in the embryonic period and is completed by $18-20$ postmenstrual weeks ${ }^{2}$. This structure is plays an esential role in the integration of motor, sensory, and cognitive functions in the human brain. The prevalence of a partial or complete agenesis of the corpus callosum in the general population is $0.1-$ $0.7 \%\left(\right.$ ref. $\left.^{3}\right)$, it is thus one of the relatively common brain anomalies. Agenesis of corpus callosum can be caused by genetic, infectious, vascular, or toxic factors ${ }^{4}$. It is reported in approximately 1:4000-1:5000 in live births but higher rates $(2-3 \%)$ have been reported among patients assessed for neurodevelopmental disorders ${ }^{5,6}$.

Ultrasound imaging is the main method for the evaluation of fetal brain development. Its valuable features are safety, economy, real-time and reproducibility. Currently, the diagnosis of corpus callosum agenesis is based mainly on two-dimensional (2D) ultrasound examination ${ }^{7}$. Image quality can be influenced by many factors such as fetal position, BMI of the patient, gestational week, physician's experience, or the amount and quality of the amniotic fluid. Detection of corpus callosum agenesis in the axial plane is based only on indirect signs such as the absence of the cavum septi pellucidi, changes in lateral ventricles, the expansion of the third ventricle and the echo between the lateral ventricle and fal ${ }^{8,9}$. However, these indirect signs are not always present at the time of the anatomy scan and may progress to be apparent in the later stages of pregnancy. Direct evaluation of the corpus callosum is not officially recommended as a part of the primary anatomy scan routine ${ }^{10}$.

Three-dimensional (3D) ultrasound imaging has emerged in recent years, enabling the acquisition of an entire volume of spatial ultrasound information that can 
be examined in real time or stored for offline analysis ${ }^{11}$. This technique allows the simultaneous presentation of the examined structure in all 3 orthogonal planes ${ }^{1}$. One of the most important views during examination of the fetal brain is the sagittal plane which provides unique information on intracranial structures such as the corpus callosum and cerebellar vermis. This scanning plane is, however, very difficult to obtain by $2 \mathrm{D}$ ultrasound ${ }^{1}$.

The aim of this study was to assess if it was beneficial to implement 3D ultrasound imaging of the fetal brain into the routine 2D ultrasound second trimester anomaly scan. An additional aim of this study was to find maternal features that could play an important role for the quality of ultrasound imaging.

\section{MATERIALS AND METHODS}

This prospective observational study was conducted in the Fetal Medicine Center of the Department of Obstetrics and Gynecology of the University Hospital Olomouc between years 2017-2020. The study population comprised of 451 consecutively scanned singleton pregnancies during routine anomaly scan at 20-22 gestational weeks. Pregnancies affected by chromosomal or structural anomalies were excluded. The 3D ultrasound imaging was performed by a single sonographer.

Transabdominal 3D ultrasound volume acquisition of the fetal brain was performed using a single designated Voluson E 10 machine, equipped with a 4-8 MHz transabdominal 3D transducer. The 3D technique used to obtain an ultrasound can be described by three main elements: 1. The section that is used to start the acquisition of the volume, 2. The angle of rotation of the mechanical sweep, and 3 . The quality of the acquisition that can be varied by the operator and depends on the number of sections obtained during acquisition. The angle of rotation and the quality of the volume influence the acquisition time and if this is too long, the probability of artifacts caused by fetal movements increases ${ }^{6}$. The multiplanar mode is the most frequently used one for imaging of fetal brain ${ }^{12-15}$

A transabdominal 3D ultrasound volume was aquired from the axial plane. The block was captured with midline of the brain orientated obliquely, at an angle 10-30 degrees from the horizontal plane with cerebellum being imaged in the closer field of the probe. Datasets from sagittal plane were acquired through the acoustic window of the metopic suture, anterior fontanel or the sagittal suture. All volumes were stored on hard disk and offline analysis was conducted using dedicated software (4D View, GE). The sonographer was instructed not to extend time of examination, that should last a maximum 30 min dedicated for complete routine anomaly scan. Initially, a gray scale volume of the fetal brain was acquired. The most important plane is the sagittal one, which provides the exact information of midline intracranial structures such as corpus callous and cerebellar vermis. This scanning plane is usually difficult to obtain according to unfavorable position of the fetus. Gentle manipulation of fetal head by free hand of the examiner was used to perform access to the midline intracranial structures and to avoid acoustic shadow from the frontal bones. The image was enlarged to at least a third of the screen. The acquisition started when both structures, i.e. corpus callosum and vermis of cerebellum, were well visualized. In case the examinator had to use both hands to improve position of the fetus, the acquisition was activated by a foot switch. If there was a problem with position of the fetus, we used virtual sagittal plane from axial acquisition. As a second step we assessed the transverse view of the fetal head at the level of the transcerebellar axial plane. This volume was easier to obtain, transcerebellar plane is the same one as during a routine second trimester screening by the $2 \mathrm{D}$ ultrasound.

The quality of images was assessed with a 5-point scale in each plane: 1 . Corpus callosum and vermis cerebella not detected; 2. Partial detection of corpus callosum or vermis cerebella; 3 . Corpus callosum fully detected; 4 . Vermis cerebella fully detected; 5 . Both structures fully detected.

\section{Statistical analyses}

All the statistical analyses were performed in the R software, version 3.6.3 (R Foundation for Statistical Computing, Vienna, Austria). For descriptive characteristics of the data, frequencies, percentages, means, and standard deviations (sd) were used. The normality of the data was assessed visually via the histograms and tested with the Shapiro-Wilk test. To compare the scale variables between groups, the Wilcoxon rank-sum and the KruskallWallis test with a Bonferroni correction for multiple group testing was used. Comparison of categorical variables was conducted with the Pearson $\chi^{2}$ test. Binary logistic regression was used to assess the predictors of quality of imaging. Significance level was set to $P<0.05$ for all statistical significance tests.

\section{RESULTS}

A total of 451 normal pregnancies at median gestational age of 20 weeks and 5 days were examined. The number of examined patients increased in time: There were $65(14.4 \%)$ pregnancies tested in years 2017-2018, $84(18.6 \%)$ in year 2019, and $302(67.0 \%)$ in year 2020 . The descriptive characteristics of the studied sample are presented in Table 1. Both the corpus callosum and vermis cerebella were detected in over a half of the examinations $(51.7 \%)$ in the sagittal plane, and almost a third (31.7\%) in the axial plane. In almost two thirds of the examinations $(61.9 \%)$, at least a partial detection in both the planes was possible. Both the structures (corpus callosum and vermis cerebelli) were fully detected in both the sagital and axial planes in $18.6 \%$ of the examinations.

Table 1 also presents comparison of the characteristics of women with different quality of imaging in the sagittal and axial planes. The mean maternal age of the tested women was 29.6 years $( \pm 5.3$ years). The maternal age was not significantly associated with the quality of imaging neither in the sagital plane $(\mathrm{H}(4)=6.33, P=0.18)$ 
Table 1. Descriptive characteristics of the sample and nonparametric comparison of medians between groups (Wilcoxon rank-sum and Kruskal-Wallis tests with Bonferroni correction for multiple comparison).

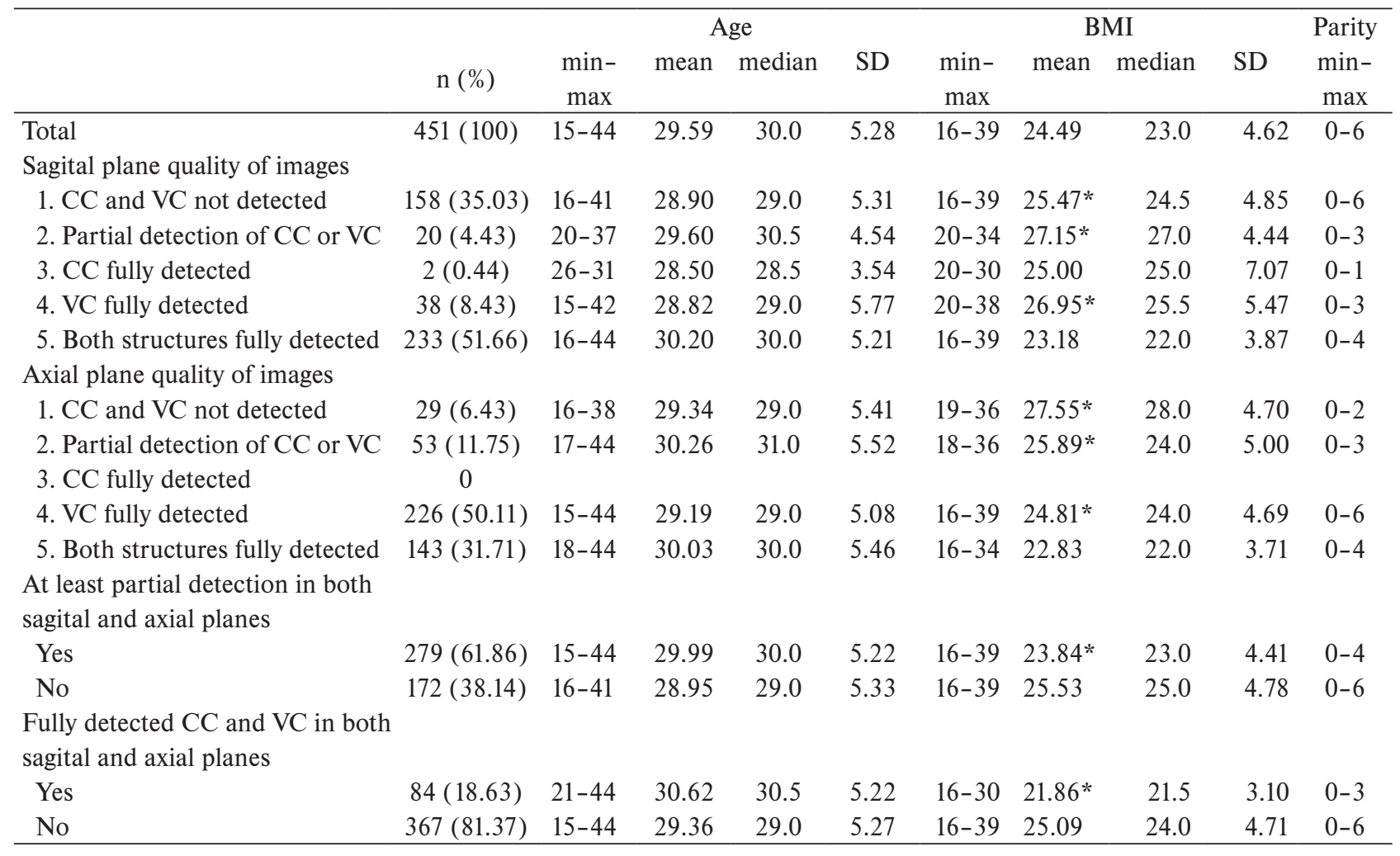

SD, standard deviation; CC, corpus callosum; VC, vermis cerebella; *Statistically significant difference $(P<0.05)$ between group 5 and other groups in the quality of images, and between groups "Yes" and "No".

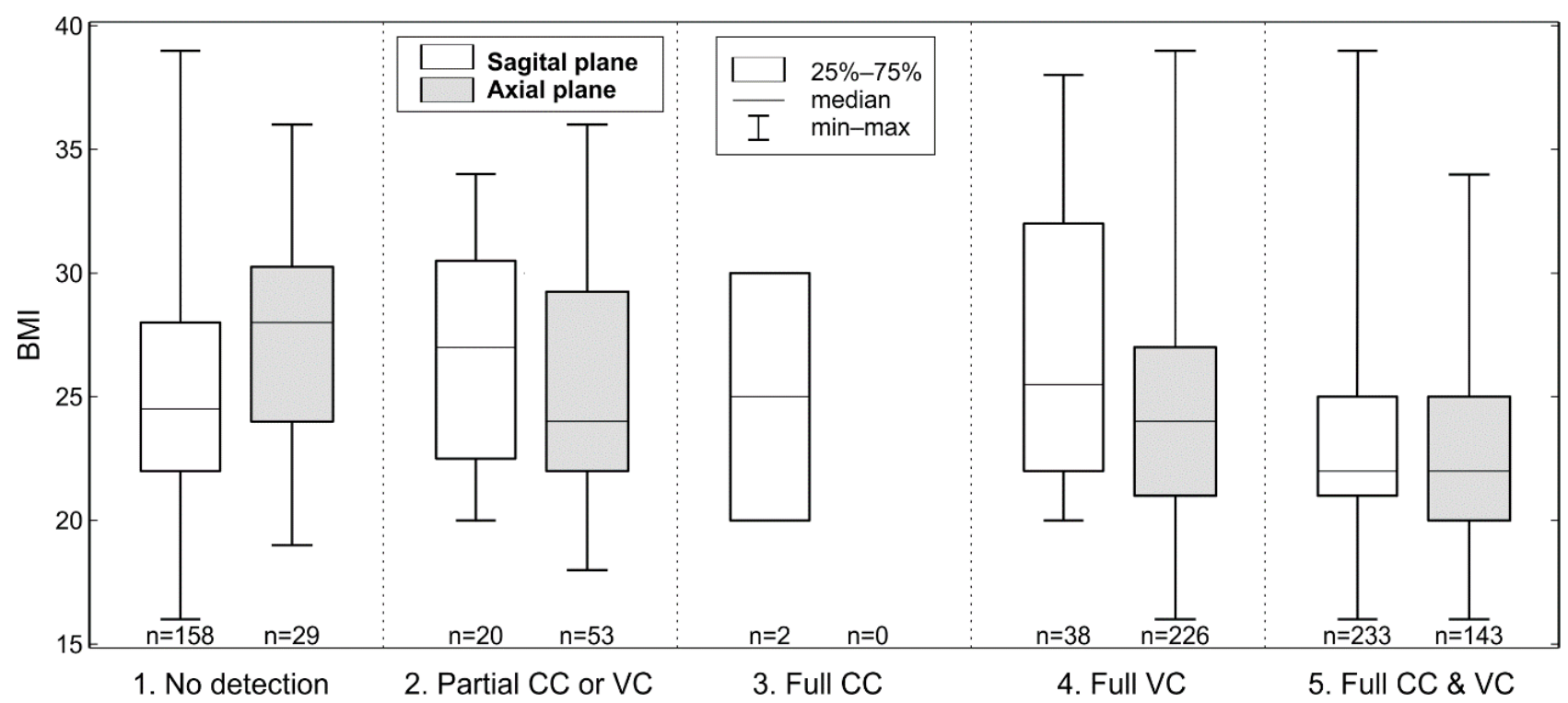

Fig. 1. Visualization of the association of BMI and quality of imaging in the sagital and axial planes.

$\mathrm{CC}$, corpus callosum; VC, vermis cerebella.

nor in the axial plane $(\mathrm{H}(3)=3.72, P=0.29)$. The mean BMI of the women was $24.5( \pm 4.6)$. BMI was found to be a significant predictor of the quality of imaging in both the sagittal plane $(\mathrm{H}(4)=40.89, P<0.001)$ and in the axial plane $(\mathrm{H}(3)=38.26, P<0.001)$. Visualization of the association of BMI and quality of imaging is presented in Fig. 1. The odds of at least partial detection of corpus callosum or vermis cerebella in both planes increased with each unit of decrease in BMI (OR=1.08, 95\% CI: 1.04-1.13, $P<0.001)$. The odds of fully detected corpus callosum and vermis cerebella in both planes significantly increased with each unit of decrease in BMI as well $(\mathrm{OR}=1.25$, 95\% CI: $1.15-1.35, P<0.001)$. The parity of the examined women was not significantly associated with the quality of imaging. 


\section{DISCUSSION}

The aim of this study was to assess the options for future implementing of 3D ultrasound imaging of the fetal head as a part of the routine second trimester screening. Another aim of the study was to assess predictors that could affect the quality of the imaging.

Our results show that the 3D imaging can be easily implemented in the routine screening. We were able to examine at least one of the studied structures in almost all cases in axial plane. Only in a third of patients were we able to display corpus callosum as a single commashaped sonolucent structure, delineated superiorly by an echogenic line. Sagittal acquisition through anterior fontanel was possible in aproximately two thirds of patients. If displayed, we could see both structures in high quality in about half of patients. It was mainly because it is difficult to obtain adequate sagittal acquisition in fetuses in the vertex presentation. We were not able to change their position during the scan period and get sagittal access through sutures. If there was a problem with position of the fetus, we used virtual sagittal plane from axial acquisition. In this case, display of corpus callosum is quite difficult and usually does not allow differentiation between the corpus callosum and the inferior cavum septi pellucidi. Most frequently it can be seen as a single comma-shaped sonolucent structure, delineated superiorly by an echogenic line. Only rarely, in good visualization conditions is the corpus callosum seen as a sonolucent stripe inserted between the inferior cavum septi pellucidi and the superior midline ${ }^{9}$. Fetuses in the breech presentation enabled easy acquisition in all planes.

The quality of $3 \mathrm{D}$ imaging is comparable to the $2 \mathrm{D}$ imaging and additionally it can display structural anomalies in multiple planes better than does the traditional 2D ultrasound ${ }^{16,17}$. 3D ultrasound was shown to be helpful in the detection of brain anomalies, especially in the midline brain structures and the posterior fossa ${ }^{8-11,18,19-25}$. Transverse view of fetal head gives us good information about vermis of cerebellum but less about corpus callosum. Visualization of posterior fossa is affected by acoustic shadow of the skull base. It can be improved by setting the angle of acquisition of about 45 degree between the incident ultrasound beam and the midline.

Associated anomalies in fetuses with the prenatal diagnosis of enlarged posterior fossa (PF) are Dandy-Walker malformation with it's prevalence of about 1 in 30,000 births; megacisterna magna with unknown prevalence; Blake's pouch cyst with prevalence 1 in 1,000 births; isolated vermian hypoplasia and vermian agenesis have unknown prevalence; $\mathrm{PF}$ arachnoid cyst has prevalence 1 in 100 births; and cerebellar hypoplasia. An enlarged PF requires specific diagnoses for the best possible counseling. The term "Dandy-Walker variant" should not be used anymore. Isolated megacisterna magna and Blake's pouch cyst can either resolve or be normal variants, but may also indicate the presence of a more severe anomaly or associated malformations ${ }^{21}$. Fetuses with enlarged posterior fossa can be detected by prenatal ultrasound screening. Diagnostic criteria have changed, and exact classification remains a challenge. Diagnostic abilities (volume ultrasound, MRI) have improved, but ultrasound and MRI may be discordant ${ }^{18,19}$.

The transabdominal 3D reconstruction of the fetal head can provide detailed evaluation of the fetal brain anatomy ${ }^{26}$. This study was planned in connection with a pilot project of Frisova et al. ${ }^{27}$ who have assessed which $3 \mathrm{D}$ technique for acquisition and post-processing datasets of the fetal brain would be the easiest and the most reliable. In their study, Frisova et al. concluded that the best technique for visualization of both the corpus callosum and vermis of cerebellum in a single image involved 3D acquisition in a sagittal suture with post-processing using 3D OVIX reconstruction. Acquisition of data was easier in a sagittal section through anterior fontanel, and these images scored second highest in the assessment of quality. Inclusion of other sagittal views would allow imaging of the structures with adequate quality in $90 \%$ of cases ${ }^{27}$. Frisova et al. concluded that acquiring 3D volumes in the sagittal plane would bring higher effectiveness of screening than acquiring precise $2 \mathrm{D}$ midsagittal sections. The 3D technique can be applied "real time" during completing the anomaly scan. It does not require extensive time for later off-line processing which often brings an immense time burden in clinical practice.

After finding the most appropriate technique for the routine screening by Frisova et al. ${ }^{27}$, our present study attempted to answer the question whether this technique was implementable as a part of the routine second trimester scan provided by a lower-level skill operator. The results of this study show that the sonographer was able to examine at least one of the studied structures in almost all cases in axial plane, and in a high proportion there was at least partial detection in both planes. Moreover, according to a subjective assessment of the sonographist, using the 3D imaging enabled to improve diagnostic quality in all the examined participants. Plasensia et al. ${ }^{28}$ used a $3 \mathrm{D}$ transabdominal ultrasound in 150 fetuses in the 20th23rd week of gestational age and found that fetal corpus callosum could easily be obtained in the fetus facing to the probe, exhaling a hypoechoic band strip. It was also demonstrated that the display rate of the corpus callosum was also different after the corpus callosum was reconstructed through different angles and planes ${ }^{28}$. Vinals et al. ${ }^{29}$ obtained a clear corpus callosum sonogram with frontal suture as the translucent window and the use of tomography ultrasound imaging. The display rate and clarity of the corpus callosum were all higher compared with the other abdominal 3D methods ${ }^{29}$. Pilu et al. ${ }^{11}$ found that the 2D views were of superior quality, in that they always allowed identification of the corpus callosum as a thin sonolucent strip with well defined echogenic contours overlying the cavum septi pellucidi. In 3D median planes, the corpus callosum could not be clearly differentiated from the inferior cavum septi pellucidi. A single comma shaped sonolucenstructure was seen, outlined superiorly by an echogenic line that in multiplanar analysis could be identified as the lower extremity of the midline echo $^{11}$. A useful complementary method to a real time 
2D ultrasound could be an off-line analysis of 3D volume data sets ${ }^{25}$

Age of the mother and her parity did not affect the quality of the 3D imaging. BMI was found to be the only significant predictor of the quality of imaging in both the sagital plane and in the axial plane. High BMI values before and during pregnancy are associated with several adverse conditions for the mother as well as the baby, e.g. gestational hypertension, diabetes, pre eclampsia, depression, a greater risk of preterm birth ${ }^{30,31}$. A systematic review $^{30}$ also reported a higher risk of foetal defects in women with higher BMI. Higher risk of defects of the fetus together with worsened quality of imaging during the pregnancy screening may produce discomfort equences for pregnant women with high BMI values. It is important to reduce the risk of adverse maternal and fetal outcomes caused by high BMI. The information about the risk connected to high BMI values is important when counselling women planning a pregnancy. Women with high BMI should be supported in losing weight before they become pregnant, and adviced to reduce the weight gain during their pregnancy.

\section{CONCLUSION}

This study has shown that 3D acquisition of fetal brain is beneficial in assessment of the corpus callosum using primarily the sagittal plane and is able to give us important information on the posterior fossa. A clear advantage of this technique, compared with the 2D ultrasound imaging, is that the fetal brain can be sequentially sectioned in all three parallel orthogonal planes ${ }^{12}$. 3D ultrasound enables off-line analysis. We can construct planes that may be difficult to obtain in $2 \mathrm{D}$ imaging. The information about the risk connected to high BMI values is important when counselling women planning a pregnancy.

Acknowledgement: I would like to thank Dr. Frisová for her help with conceptualization, visualization, methodology settings and editing the article.

Author contributions: MMT, ML: conceptualization; MMT, ML, JF: methodology; JF: data analysis, visualization; MMT, JF: original draft preparation; ML: review and editing; ML: supervision; All authors have read and agreed to the published version of the manuscript.

Conflict of interest statement: The authors state that there are no conflicts of interest regarding the publication of this article.

\section{REFERENCES}

1. International Society of Ultrasound in Obstetrics \& Gynecology Education Committee. Sonographic examination of the fetal central nervous system: guidelines for performing the basic examination and the fetal neurosonogram. Ultrasound Obstet Gynecol 2007;29:109-16.

2. Schell-Apacik CC, Wagner K, Bihler M, Ertl-Wagner B, Heinrich U, Klopocki E, Kalscheuer VM, Muenke M, von Voss H. Agenesis and dysgenesis of the corpus callosum: clinical, genetic and neu- roimaging findings in a series of 41 patients. Am J Med Genet A 2008;146A(19):2501-11. doi:10.1002/ajmg.a.32476

3. Grogono JL. Children with agenesis of the corpus callosum. Dev Med Child Neurol 1968;10:613-16.

4. Paul LK, Brown WS, Adolphs R, Tyszka JM, Richards LJ, Mukherjee $P$, Sherr EH. Agenesis of the corpus callosum genetic, developmental and functional aspects of connectivity. Nat Rev Neurosci 2007;8:28799.

5. Wuest A, Surbek D, Wiest R, Weisstanner Ch, Bonel H, Steinlin M, Raio $L$, Tutschek B. Enlarged posterior fossa on prenatal imaging: differential diagnosis, associated anomalies and postnatal outcome. Nordic Federation of Societies of Obstetrics and Gynecology, Acta Obstetricia et Gynecologica Scandinavica 2017;96:837-43.

6. D’Antonio F, Khalil A, Garel C, Pilu G, Rizzo G, Lerman-Sagie T, Bhide A, Thilaganathan B, Manzoli L, Papageorghiou AT. Systematic review and meta-analysis of isolated posterior fossa malformations on prenatal imaging (part 2): neurodevelopmental outcome. Ultrasound Obstet Gynecol 2016;4828-37.

7. Falco P, Gabrielli S, Visentin A, Perolo A, Pilu G, Bovicelli L. Transabdominal sonography of the cavum septum pellucidum in normal fetuses in the second and third trimesters of pregnancy. Ultrasound Obstet Gynecol 2000;16: 549-553.

8. Malinger G, Lev D, Kidron D, Heredia F, Hershkovitz R, LermanSagie T. Differential diagnosis in fetuses with absent septum pellucidum. Ultrasound Obstet Gynecol 2005;25:42-49.

9. Laskin MD, Kingdom J, Toi A, Chitayat D, Ohlsson A. Perinatal and neurodevelopmental outcome with isolated fetal ventriculomegaly: a systematic review. J Matern Fetal Neonatal Med 2005;18:289-98.

10. Li Y, Tao GW, Pan MZ, Shao GR. Comparison of traditional 2D and 3D Omniview technique in detection of fetus corpus callosum. Med Ultrason 2018;20(4):493-97. doi: 10.11152/mu-1555

11. Pilu G, Segata M, Ghi T, Carletti A, Perolo A, Santini D, Bonasoni P, Tani $\mathrm{G}$, Rizzo N. Diagnosis of midline anomalies of the fetal brain with the three-dimensional median view. Ultrasound Obstet Gynecol 2006;27(5):522-29.

12. Correa FF, Lara C, Bellver J, Remohi J, Pellicer A, Serra V. Examination of the fetal brain by transabdominal three-dimensional ultrasound: potential for routine neurosonographic studies. Ultrasound Obstet Gynecol 2006;27:503-8.

13. Kalache KD, Eder K, Esser T, Proquitte H, Stoltenburg-Didinger G, Hartung JP, Bamberg C. Three-dimensional ultrasonographic reslicing of the fetal brain to assist prenatal diagnosis of central nervous system anomalies. J Ultrasound Med 2006;25:509-14.

14. Timor-Tritsch IE, Monteagudo A, Mayberry P. Three-dimensional ultrasound evaluation of the fetal brain: the three horn view. Ultrasound Obstet Gynecol 2000;16:302-6.

15. Tonni G, Grisolia G, Sepulveda W. Second trimester fetal neurosonography: reconstructing cerebral midline anatomy and anomalies using a novel three-dimensional ultrasound technique. Prenat Diagn 2014;34:75-83.

16. Glass HC, Shaw GM, Ma C, Sherr EH. Agenesis of the corpus callosum in California 1983-2003: a population-based study. Am J Med Genet A 2008;146A:2495-500.

17. Tortori-Donati P, Fondelli MP, Rossi A, Carini S. Cystic malformations of the posterior cranial fossa originating from a defect of the posterior membranous area. Megacisterna magna and persisting Blake's pouch: two separate entities. Childs Nerv Syst 1996;12:303-8.

18. Bornstein E, Monteagudo A, Santos R, Keeler SM, Timor-Tritsch IE. A systematic tech- nique using 3-dimensional ultrasound provides a simple and reproducible mode to evaluate the corpus callosum. Am J Obstet Gynecol 2010;202(2):201.e1-5. doi: 10.1016/j. ajog.2009.10.705

19. Monteagudo A, Timor-Tritsch IE, Mayberry P. Three-dimensional transvaginal neuro- sonography of the fetal brain: "navigating" in the volume scan. Ultrasound Obstet Gynecol 2000;16:307-13.

20. Vinals F, Munoz M, Naveas R, Shalper J, Giuliano A. The fetal cerebellar vermis: anatomy and biometric assessment using volume contrast imaging in the C-plane ( $\mathrm{VCl}-\mathrm{C})$. Ultrasound Obstet Gynecol 2005;26:622-27.

21. Paladini D, Volpe P. Posterior fossa and vermian morphometry in the characterization of fetal cerebellar abnormalities: a prospective three-dimensional ultrasound study. Ultrasound Obstet Gynecol 2006;27:482-89.

22. Zalel Y, Yagel S, Achiron R, Kivilevich Z, Gindes L. Three dimensional 
ultrasonography of the fetal vermis at 18 to 26 weeks' gestation: time of appearance of the primary fissure. J Ultrasound Med 2009;28:1-8.

23. Hata T, Yanagihara T, Matsumoto M, Hanaoka U, Ueta M, Tanaka $Y$, Kanenishi K, Kuno A, Yamashiro C, Ohnishi Y, Tanaka H, Hayashi K. Three-dimensional sonographic features of fetal central nervous system anomaly. Acta Obstet Gynecol Scand 2000;79:635-39.

24. Salman MM, Twining $P$, Mousa $H$, James $D$, Momtaz $M$, Aboulghar $\mathrm{M}$, El-Sheikhah A, Bugg GJ. Evaluation of offline analysis of archived three-dimensional volume datasets in the diagnosis of fetal brain anomalities. Ultrasound Obstet Gynecol 2011;38:165-69.

25. Szabo N, Gergev G, Kobor J, Bereg E, Turi S, Sztriha L. Corpus callosum anomalies: birth prevalence and clinical spectrum in Hungary. Pediatr Neurol 2011;44:420-6.

26. Ghi T, Casadio P, Kuleva M, Perrone AM, Savelli L, Giunchi S, Meriggiola MC, Gubbini, G, Pilu G, Pelusi, C, Pelusi G. Accuracy of three-dimensional ultrasound in diagnosis and classification of congenital uterine anomalies. Fertil Steril 2009;92:808-13.
27. Frisova, V, Srutova M, Hyett J. 3-D Volume Assessment of the Corpus Callosum and Cerebellar Vermis Using Various Volume Acquisition and Post-Processing Protocols. Fetal Diagn Ther 2018;43(3):199-207.

28. Plasencia W, Dagklis T, Borenstein M, Csapo B, Nicolaides KH. Assessment of the corpus callosum at 20-24 weeks' gestation by three-dimensional ultrasound examination. Ultrasound Obstet Gyncol 2007;30(2):169-72.

29. Vinals F, Munoz M, Naveas R, Giuliano A. Transfrontal three-dimensional visualization of midline cerebral structures.Ultrasound Obstet Gynecol 2007;30(2):162-68.

30. Marchi J, Berg M, Dencker A, Olander EK, Begley C. Risks associated with obesity in pregnancy, for the mother and baby: a systematic review of reviews. Obesity Reviews 2015;16(8): 621-38.

31. Torloni MR, Betran AP, Horta BL, Nakamura MU, Atallah AN, Moron $\mathrm{AF}$, Valente $\mathrm{O}$. Prepregnancy $\mathrm{BMI}$ and the risk of gestational diabetes: a systematic review of the literature with meta-analysis. Obesity reviews 2009;10(2):194-203. 